\title{
A Cost Effective Virtual Cluster with Hadoop Framework for Big Data Analytics
}

\author{
Seraj Al Mahmud Mostafa ${ }^{1}$ and A.B.M Moniruzzaman \\ Member, IEEE, ${ }^{2}$ Department of Computer Science and Engineering \\ Daffodil International University \\ Dhaka, Bangladesh \\ sammbd@gmail.com ${ }^{1}$ \\ abm.moniruzzaman.eng@ieee.org ${ }^{2}$
}

\begin{abstract}
Big data processing is currently becoming increasingly important research field in computer technology professionals due to the continuous growth of the amount of data generated by various fields. However, the processing of large-scale research data requires cluster technology infrastructure which causes huge investments for educational institutions. Hadoop is an open-source framework that allows for distributed storage and processing of very large data sets on computer clusters built from commodity hardware. This technology is being widely used for the analysis of large datasets. This paper focuses on proposes a low cost scalable hadoop virtual cluster platform and the performance of hadoop irtual cluster. We first describe the design and implementation of a virtual datacenter using hadoop framework. Then we perform a set of experiments to investigate the performance of virtual datacenter with standard datasets. For experiment and evaluation, the Cloudera's distribution of Apache Hadoop $(\mathrm{CDH})$ is installed and configured on the DIU Cloud and Big Data Lab as a prototype implementation of a virtual data center and tested with 40 workstations. In this paper, we use TPC BENCHMARK TM DS” by-Transaction Processing Performance Council (TPC) benchmarks for evaluation virtual data center performance. The contributions of this paper, is to design model and implement a cost effective elastic virtual data center with hadoop framework and resource utilizations for educational institutions to provide high performance for distributed and parallel processing; as well as, Identify the bottlenecks of this systems e.g. bandwidth of network connectivity with all nodes in the lab.
\end{abstract}

Keywords- Hadoop; MapReduce; Virtual Cluster; Cloud Computing; Big Data

\section{Introduction}

Big data [1-3] has recently received considerable attention to research field due to the continuous growth of the amount of data generated by various fields such as particle physics, human genomics, earth observation, etc. How to compute, transfer, and store these huge data is a prominent challenge which will bring great impact on the traditional architectures and methods of computation, networking and storage. Therefore big data becoming important and cutting edge research field in higher studies; and dig data research labs are being introducing in higher educational institutions in recent years. However, if we consider computer labs, in many educational institutes, we find so many desktop and low-end servers which are utilize only $15 \%$ to $25 \%$. Therefore, we can detect such hardware resources and use them to design a low cost virtual datacenter of distributed and parallel processing architecture, where we can process big amount of research data like structured, unstructured and semi-structured data. 


\section{Related Technologies}

\subsection{Virtualization Technology}

Virtualization technology is currently becoming increasingly popular as a core technology to implement the cloud computing paradigm. Many efforts have been made to study the performance characterization of virtualization, including performance evaluation, performance modeling, and performance optimization. Server consolidation is one of the most important application scenarios of virtualization to improve the resource utilization.

\subsection{Hadoop}

Apache Hadoop [4-6] is an open-source software framework written in Java for distributed storage and distributed processing of very large data sets on computer clusters. The system splits the input data-set into multiple chunks, each of which is assigned a map task that can process the data in parallel. Each map task reads the input as a set of (key, value) pairs and produces a transformed set of (key, value) pairs as the output. The framework shuffles and sorts outputs of the map tasks, sending the intermediate (key, value) pairs to the reduce tasks, which group them into final results. MapReduce uses JobTracker and TaskTracker mechanisms to schedule tasks, monitor them, and restart any that fail.

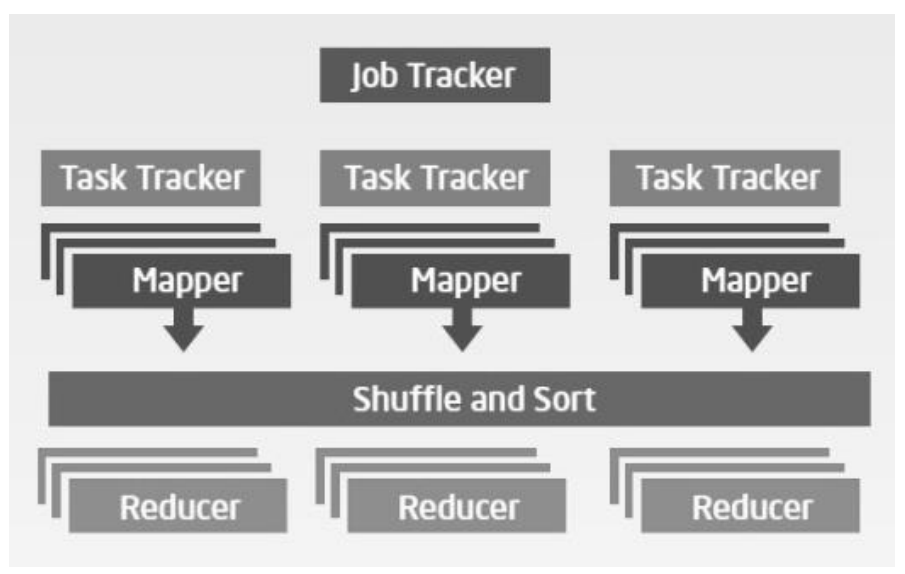

Figure 1. Logical Architecture of Hadoop

The Apache Hadoop platform also includes the Hadoop Distributed File System (HDFS) [7-8], which is designed for scalability and fault tolerance. HDFS stores large files by dividing them into blocks (usually 64 or $128 \mathrm{MB}$ ) and replicating the blocks on three or more servers. HDFS provides APIs for MapReduce [9-10] applications to read and write data in parallel. Capacity and performance can be scaled by adding Data Nodes, and a single NameNode mechanism manages data placement and monitors server availability. 


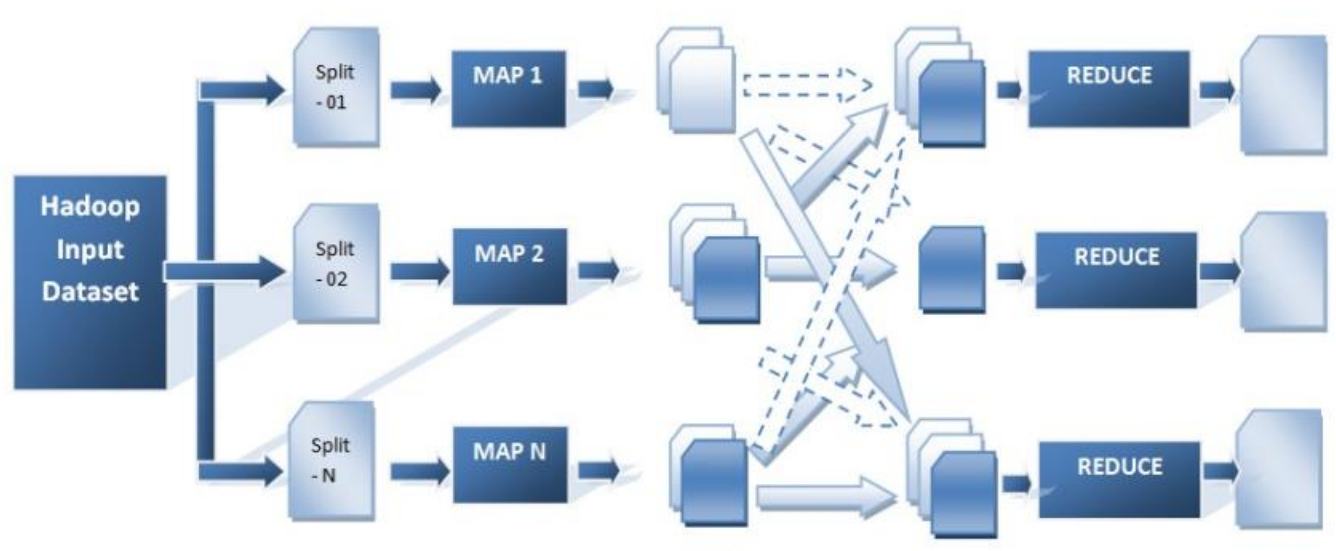

Figure 2. Hadoop Process Flow

HDFS clusters in production use today reliably hold petabytes of data on thousands of nodes. MapReduce, the programming paradigm implemented by Apache Hadoop, breaksup a batch job into many smaller tasks for parallel processing on a distributed system HDFS, the distributed file system stores the data reliably shown in the Figure 1 and Figure 2.

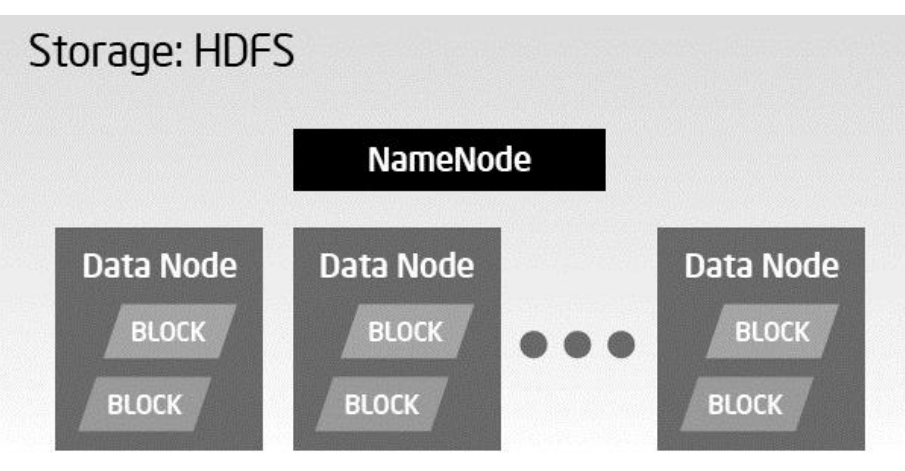

Figure 3. HDFS Storage

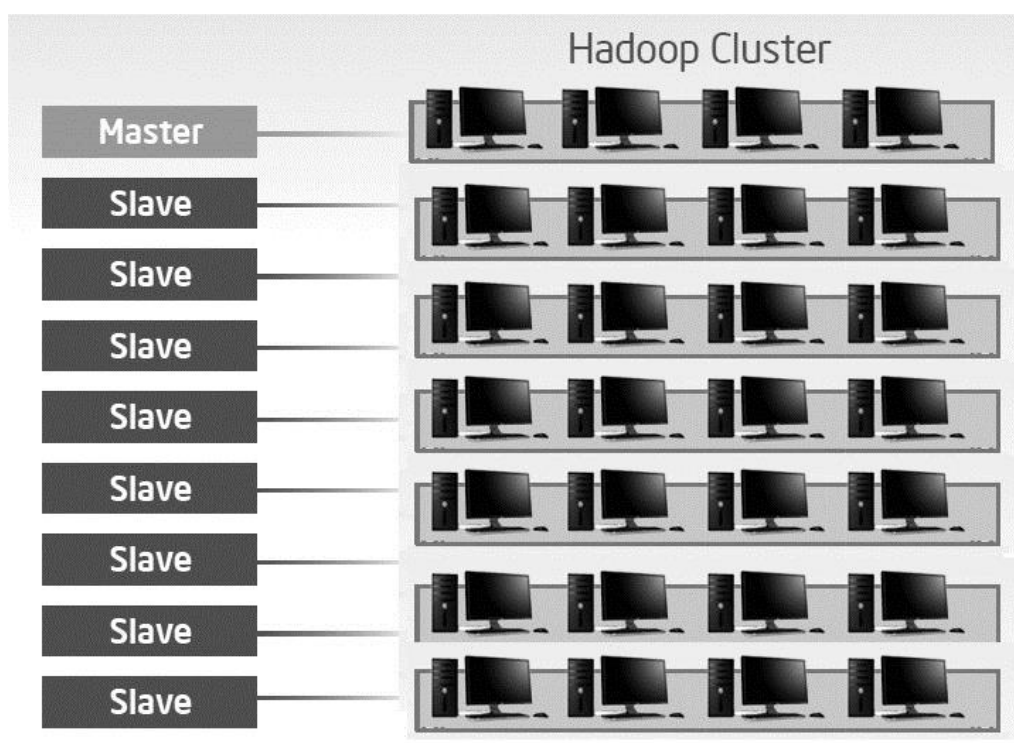

Figure 4. Physical Architecture of Hadoop Cluster 


\subsection{MapReduce}

MapReduce is a programming model and an associated implementation for processing and generating large data sets with a parallel, distributed algorithm on a cluster. A MapReduce program is composed of a Map() procedure that performs filtering and sorting (such as sorting students by first name into queues, one queue for each name) and a Reduce() procedure that performs a summary operation (such as counting the number of students in each queue, yielding name frequencies).

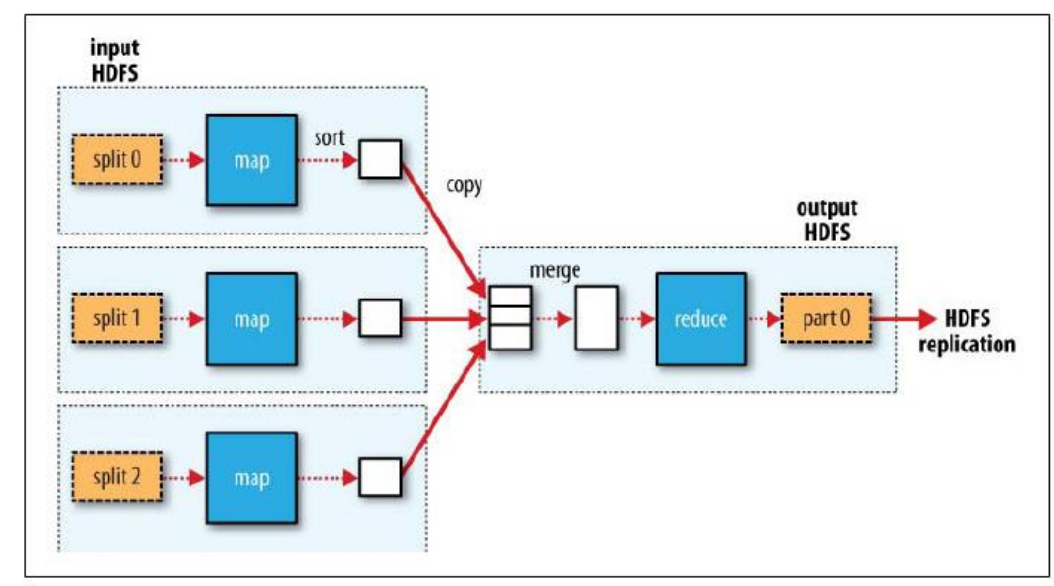

Figure 5. MapReduce Data Flow with a Single Reduce Task [6]

MapReduce optimized the hadoop provisioning in the cloud to reduce the cost and improve the performance, and compared the performance of hadoop cluster on virtual machines and physical machines and found that running MapReduce application on virtual machines incurs additional performance degradation compared to the case that running on physical machines.

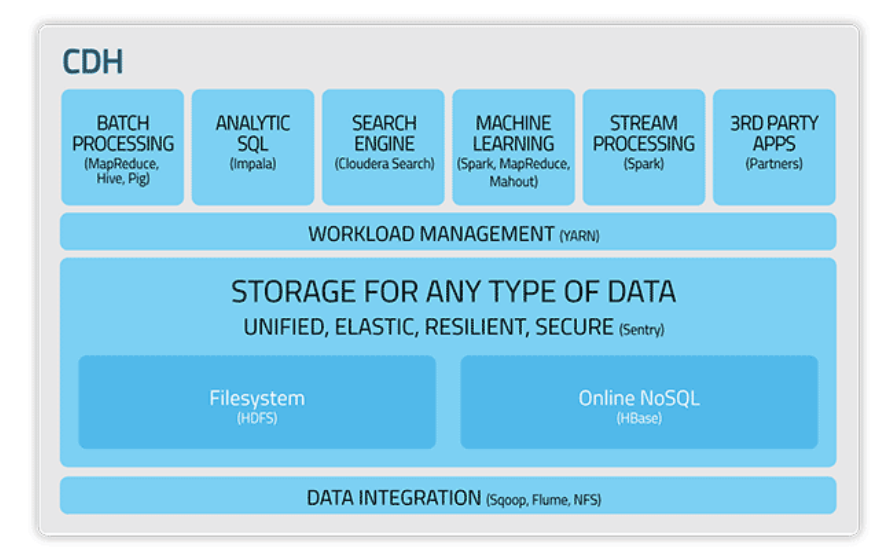

\section{Figure 6. CDH (Cloudera Distribution Including Apache Hadoop) Key Elements}

Cloudera's open-source Apache Hadoop distribution [11], CDH (Cloudera Distribution Including Apache Hadoop) [12-13] is the only Hadoop solution to offer unified batch processing, interactive SQL, and interactive search, and role-based access controls. CDH delivers the core elements of Hadoop - scalable storage and distributed computing along with additional components such as a user interface. 


\section{Implementation of Hadoop Virtual Cluster}

\subsection{Infrastructure Design}

We design and with a small lab with 40 worktsations and we separate logically two group and they have 20 workstations in each site. Now design a virtual datacenter using these 40 workstations and additional two high-end server. User can use these workstations to perform their usual purpose and in background we can install hypervisor to create another virtual machine on each physical machine. Combining those virtual machine (Slave/DataNode) and two high-end machine (Master/NameNode) with Hadoop cluster and design a virtual datacenter infrastructure with distributed and parallel processing power.

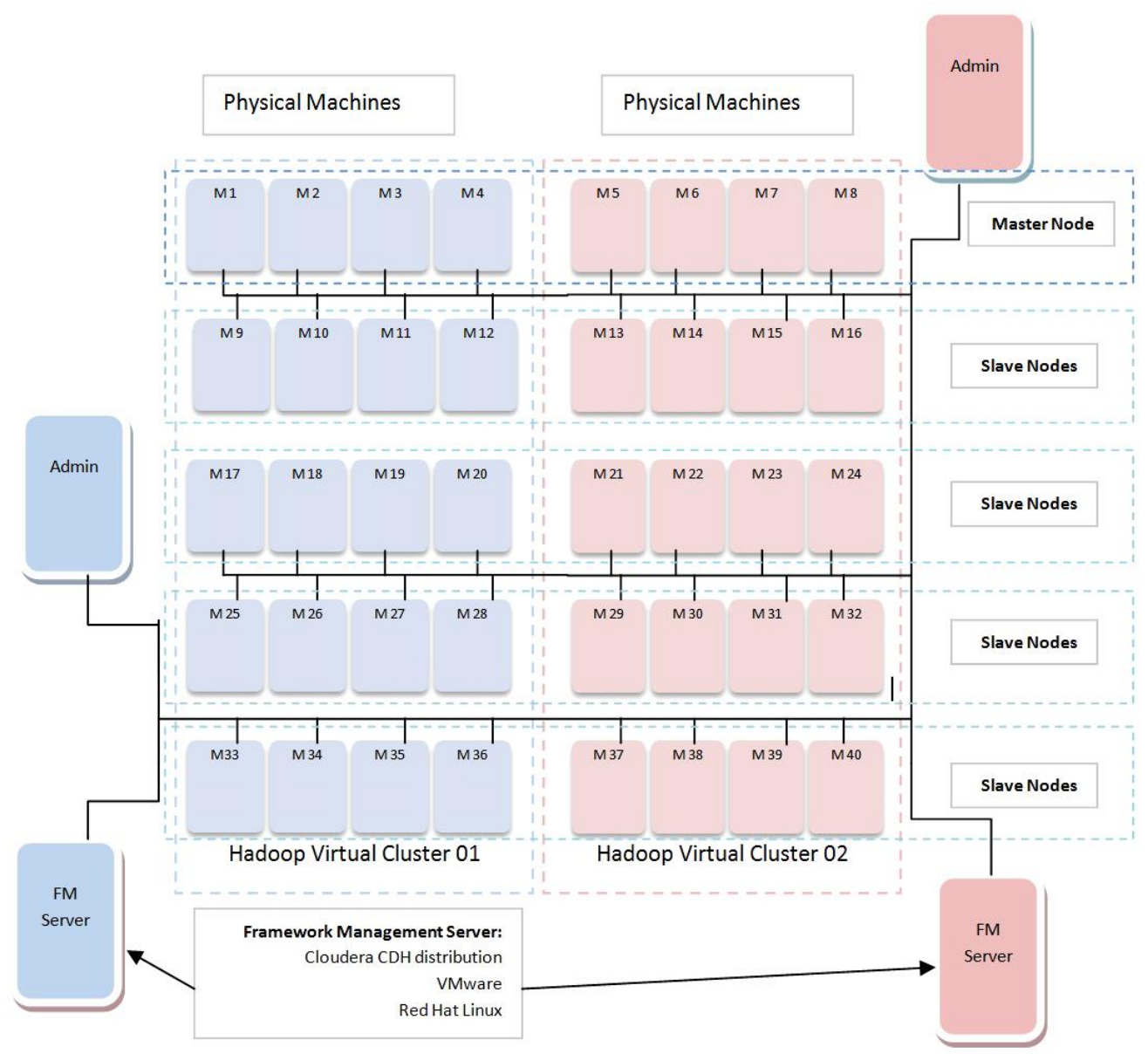

Figure 7. Infrastructure Design of a Virtual Cluster With Hadoop Framework

First of all place a high-end server in each site which is called as cluster Master/NameNode and other 20 workstation will work as Slave/DataNode. Hadoop framework will be install on Master/NameNode. Need to install Hypervisor software in each workstation to create a virtual server and add the virtual server to the Hadoop cluster as Slave/DataNode.Same task need to complete on both site and finally conFigure Hadoop cluster services and other related services to Master and Slave nodes.

In the infrastructure design in Figure 7 where two virtual cluster are connected with each other through private network. Each cluster has LAN connection and all the 
workstations are connected to LAN. A high-end server has been placed to each cluster connected to LAN.

\subsection{Environment Setup}

3.2.1. Installation and Configuration: To simulate the infrastructure 64 bit Red Hat Linux6 has been used as operating system on Master and Slave servers in this project. Cloudera Manager CDH5.2.0 distribution has been used for Hadoop cluster framework and VMware has been used as hypervisor to prepare this virtual datacenter as a simulation. Please find the installation steps and command as below.

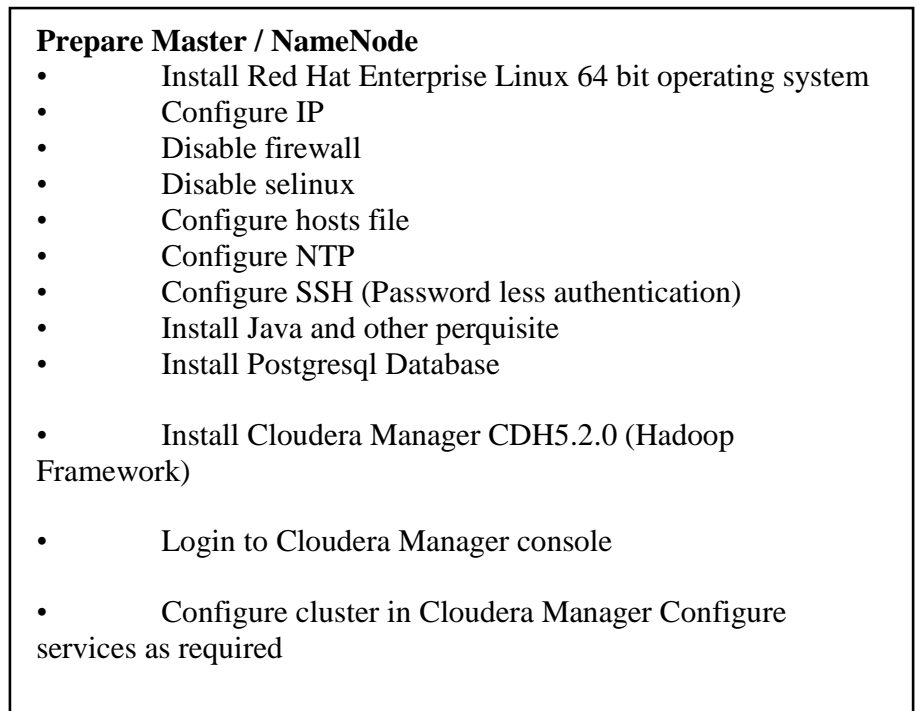

\section{Prepare Slave / Data Node}

- Install Red Hat Enterprise Linux 64 bit operating system

- $\quad$ Configure IP

- Disable firewall

- Disable selinux

- Configure hosts file

- Configure NTP

- Configure SSH (Password less authentication)

- Install Java and other perquisite

- Login to Cloudera Manager console

- Configure data node to the cluster onfioure services as renuired

\begin{tabular}{|l|l|l|}
\hline $\mathbf{1}$ & $\begin{array}{l}\text { ConFigure IP Address (Master } \\
\text { and Slave) setup }\end{array}$ & $\begin{array}{l}\text { vim/etc./sysconfig/network-scripts/ifcfg-eth, ONBOOT=yes, } \\
\text { service network restart }\end{array}$ \\
\hline $\mathbf{2}$ & $\begin{array}{l}\text { Stop Firewall (Master and } \\
\text { Slave) }\end{array}$ & $\begin{array}{l}\text { chkconfig --list iptables } \\
\text { chkconfig --level 0123456 iptables off } \\
\text { serviceiptables stop } \\
\text { serviceiptables status }\end{array}$ \\
\hline $\mathbf{3}$ & $\begin{array}{l}\text { Disableselinux (Master and } \\
\text { Slave) }\end{array}$ & $\begin{array}{l}\text { sestatus } \\
\text { vim /etc./sysconfig/selinux } \\
\text { SELINUX=disabled } \\
\text { Init 0 }\end{array}$ \\
\hline $\mathbf{4}$ & ConFigure Hostname & vim/etc./sysconfig/network \\
\hline
\end{tabular}




\begin{tabular}{|c|c|c|}
\hline & & $\begin{array}{l}\text { NETWORKING=yes } \\
\text { HOSTNAME }=\text { XXXXXXXX } \\
\text { GATEWAY }=\text { XXX.XXX.XXX.XXX } \\
\text { vim /etc./hosts } \\
\text { IP }<X X X . X X X . X X X . X X X>\text { FQDN }<X X X X . X X X X X . X X X>\end{array}$ \\
\hline 5 & $\begin{array}{l}\text { ConFigure NTP } \\
\text { (Master and Slave) }\end{array}$ & $\begin{array}{l}\text { cd letc. } \\
\text { catntp.conf |more } \\
\text { vintp.conf |more } \\
\text { cd letc./init.d } \\
\text { service ntpd status } \\
\text { service ntpd start } \\
\text { ntpq -p } \\
\text { chkconfig --list --level } 345 \text { |grepntp } \\
\text { chkconfigntpd on --level } 345 \\
\text { chkconfig --list --level } 345 \text { |grepntpd }\end{array}$ \\
\hline 6 & $\begin{array}{l}\text { ConFigure SSH for Password } \\
\text { less login (Master and Slave) }\end{array}$ & $\begin{array}{l}\text { login to slave01: } \\
\text { ssh-keygen -t rsa } \\
\text { cd /root/.ssh } \\
\text { ls } \\
\text { Login to master01: } \\
\text { ssh-keygen -t rsa } \\
\text { cd /root/.ssh } \\
\text { ls } \\
\text { cat id_rsa.pub >>authorized_keys } \\
\text { scpauthorized_keys root@slave01:/root/.ssh/ } \\
\text { login to slave01: } \\
\text { cd /root/.ssh } \\
\text { ls } \\
\text { cat id_rsa.pub >>authorized_keys } \\
\text { scpauthorized_keys root@ @master01:/root/.ssh/ } \\
\text { Test: } \\
\text { ssh slave01 (able to login without password) } \\
\text { ssh master02 (able to login without password) }\end{array}$ \\
\hline 7 & $\begin{array}{l}\text { Install Postgresql8.4 Database } \\
\text { (Master Server) }\end{array}$ & $\begin{array}{l}\text { Create folder and copy jre and postgresql software to that folder: } \\
\text { chmod } 777 \text { jre-7u1-linux-x64.tar.gz } \\
\text { tar -zxvf jre-7u1-linux-x64.tar.gz } \\
\text { cd / } \\
\text { host to export java path: export } \\
\text { PATH=\$PATH:/soft/jre1.7.0_01/bin/ } \\
\text { chmod 777 postgresq184-8.4.22-1PGDG.rhel6.x86_64.rpm } \\
\text { chmod 777 postgresq184-libs-8.4.22-1PGDG.rhel6.x86_64.rpm } \\
\text { chmod 777 postgresq184-server-8.4.22-1PGDG.rhel6.x86_64.rpm } \\
\text { rpm -Uvh postgresq184-libs-8.4.22-1PGDG.rhel6.x86_64.rpm } \\
\text { rpm -Uvh postgresq184-8.4.22-1PGDG.rhel6.x86_64.rpm } \\
\text { rpm -Uvh postgresq184-server-8.4.22-1PGDG.rhel6.x86_64.rpm }\end{array}$ \\
\hline 8 & $\begin{array}{l}\text { Check services and logs after } \\
\text { installation (Master Server) }\end{array}$ & $\begin{array}{l}\text { service postgresql-8.4 status } \\
\text { service postgresql-8.4 initdb } \\
\text { service postgresql-8.4 start } \\
\text { chkconfig --level } 345 \text { postgresql- } 8.4 \text { on } \\
\text { chkconfig --list |greppostgre* }\end{array}$ \\
\hline 9 & $\begin{array}{l}\text { ConFigure yum.conf file if } \\
\text { proxy used for internet } \\
\text { (Master and Slave) }\end{array}$ & $\begin{array}{l}\text { vim /etc./yum.conf } \\
\text { proxy=http://XXX.XXX.XXX.XXX:XXXX }\end{array}$ \\
\hline 10 & $\begin{array}{l}\text { DownloadCloudera manager } \\
\text { (Master Server) }\end{array}$ & $\begin{array}{l}\text { cd /root } \\
\text { wget http://archive.cloudera.com/cm5/installer/latest/cloudera- } \\
\text { manager-installer.bin } \\
\text { ls-1 }\end{array}$ \\
\hline 11 & $\begin{array}{l}\text { Install Cloudera } \text { Manager } \\
\text { (Master Server) }\end{array}$ & $\begin{array}{l}\text { Cd /root } \\
1 \text { s -1 } \\
\text {-rw-r--r-- } 1 \text { root root } 501703 \text { Jul } 2910: 51 \text { cloudera-manager- } \\
\text { installer.bin } \\
\text { chmod } 777 \text { cloudera-manager-installer.bin } \\
\text {./cloudera-manager-installer.bin }\end{array}$ \\
\hline
\end{tabular}


After this step cloudera manager will be installed on the Master server and go the following link (http://XXX.XXX.XXX.XXX <IP>:7180/) to install and conFigure Hadoop cluster.

\begin{tabular}{|c|c|c|}
\hline 1 & $\begin{array}{l}\text { Check services after } \\
\text { installation (Master Server) }\end{array}$ & $\begin{array}{l}\text { cd /var/log/cloudera-manager-installer } \\
\text { less 6.start-scm-server.log (check OK) } \\
\text { less 5.start-embedded-db.log (check OK) } \\
\text { chkconfig --level } 345 \text { cloudera-scm-server-db on } \\
\text { chkconfig --level } 345 \text { cloudera-scm-server on }\end{array}$ \\
\hline 2 & $\begin{array}{l}\text { Yum configuration (All Slave } \\
\text { Servers) }\end{array}$ & $\begin{array}{l}\text { cd /etc./yum.repos.d/ } \\
\text { vim server_rhel6.repo } \\
\text { [local_mirror_server_rhel6] } \\
\text { name=patch_mirror_server_rhel6 } \\
\text { baseurl=ftp://XXX.XXX.XXX.XXX/repo/rhel-x86_64-server- } \\
\text { 6/getPackage } \\
\text { enabled=1 } \\
\text { gpgcheck=0 } \\
\text { yum clean all } \\
\text { yumrepolist } \\
\text { yum list all }\end{array}$ \\
\hline 3 & $\begin{array}{l}\text { Install dependent services (All } \\
\text { Slave Servers) }\end{array}$ & $\begin{array}{l}\text { yum install cyrus-sasl-gssapi } \\
\text { yum install redhat-lsb } \\
\text { yum install portmap } \\
\text { servicerpcbind status } \\
\text { servicerpcbind start }\end{array}$ \\
\hline 4 & $\begin{array}{l}\text { ConFigure Hadoop Cluster } \\
\text { (Cloudera Manager Console) }\end{array}$ & $\begin{array}{l}\text { Go the following link (http://XXX.XXX.XXX.XXX }<\mathrm{IP}>: 7180 / \text { ) to } \\
\text { install and conFigure Hadoop cluster that is showing in the Figure } \\
2.6 \text { Default User ID and Password is admin, admin }\end{array}$ \\
\hline
\end{tabular}

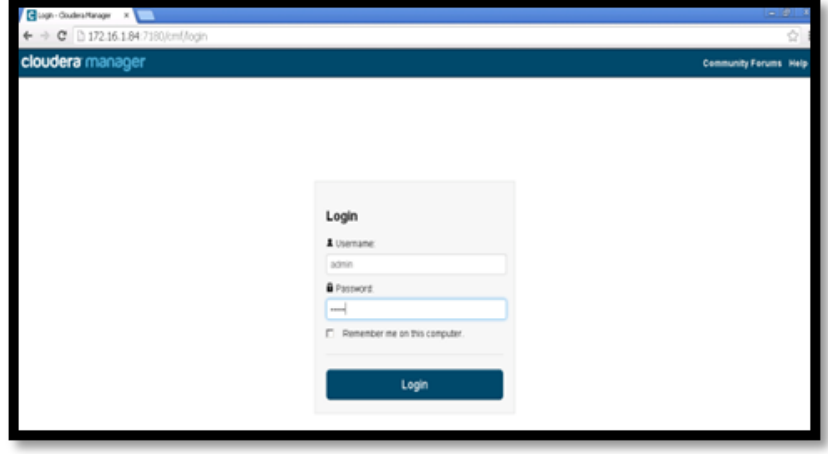

Figure 8. Cloudera Manager Console to Login

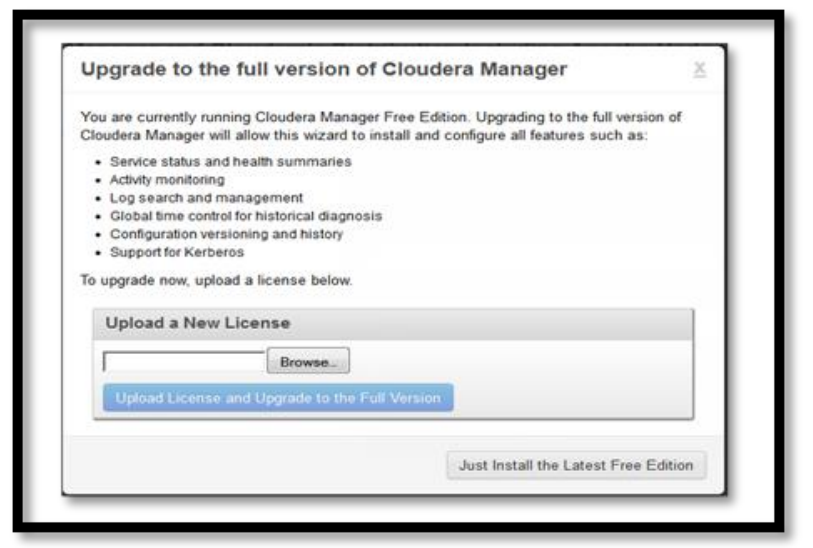

Figure 9. Cloudera Manager Version Selection 
Figure 8 showing to click on just free installation to choose the Cloudera Manager version.

Figure 10 and Figure 11 showing the how to add host to create a cluster on Cloudera manager. Add hostnames as defined in on /etc./hosts file of all servers.

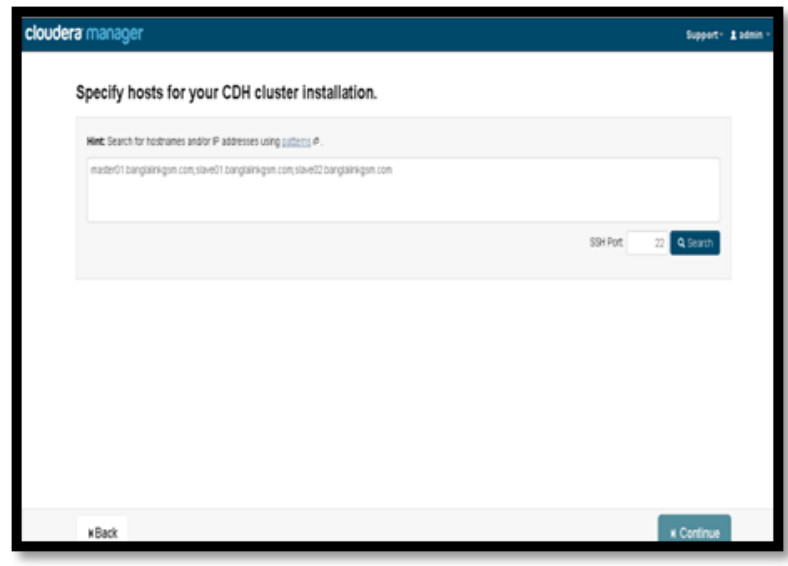

Figure 10. Cluster Installation

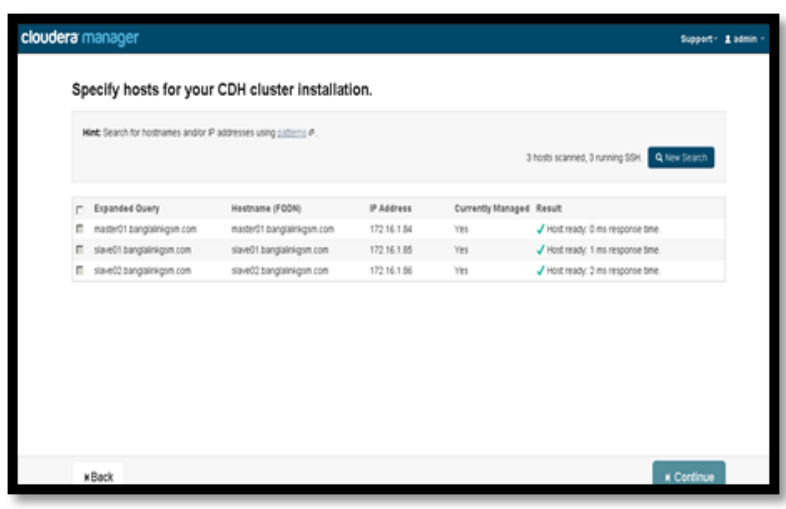

Figure 11. Specify Host to Install Cluster

Figure 11 showing the hosts availability, now select all the available hosts by checking the text box against it. Figure 12 shows the $\mathrm{CDH}$ version selection where we need to click on CDH4 cloudera manager latest version of Hadoop.

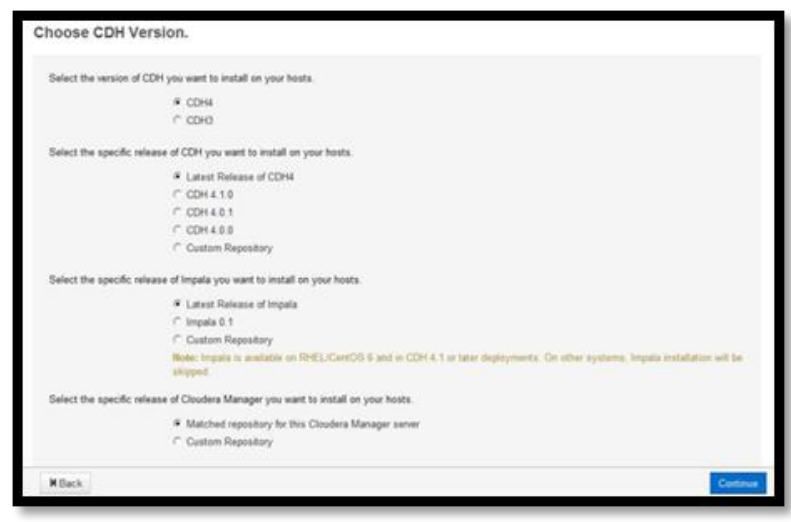

Figure 12. CDH Version Selection 


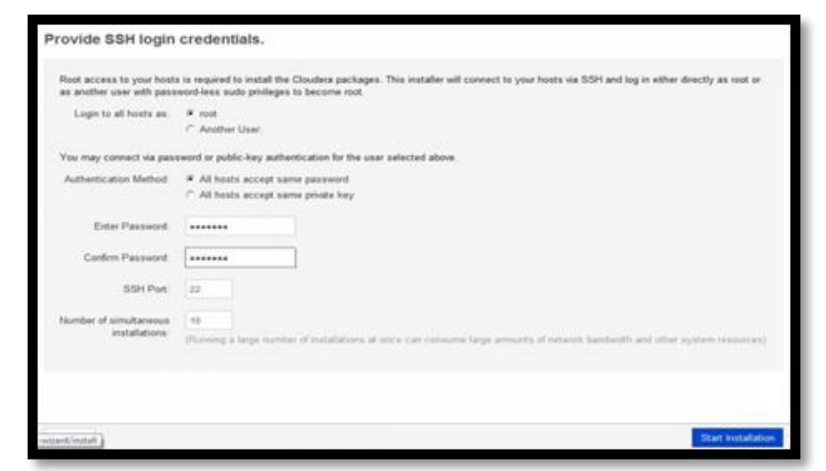

Figure 13. CDH Installation Credential Option

Figure 13 shows the installation credential option where we need to click on start installation button to start the installation process on selected three nodes. Figure 14 shows this process is successfully completed click on continue button and that will run host inspector to check the host for correctness. After the inspector is finished click on continue

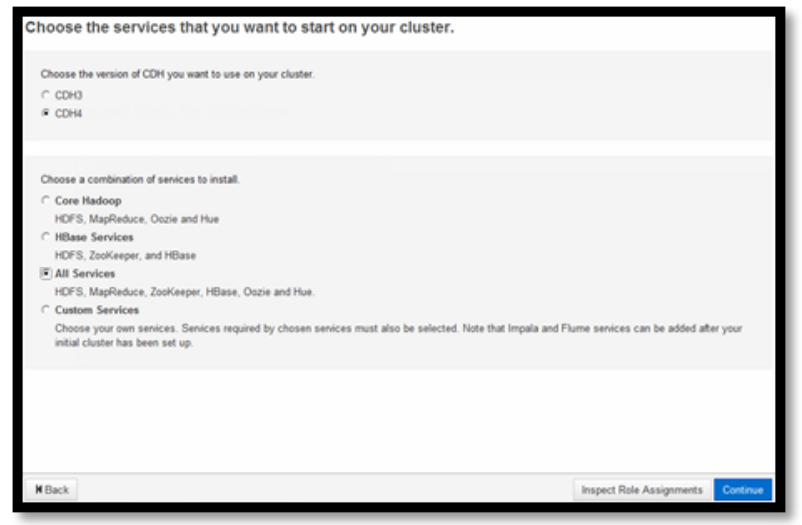

Figure 14. Inspect Hosts for Correctness

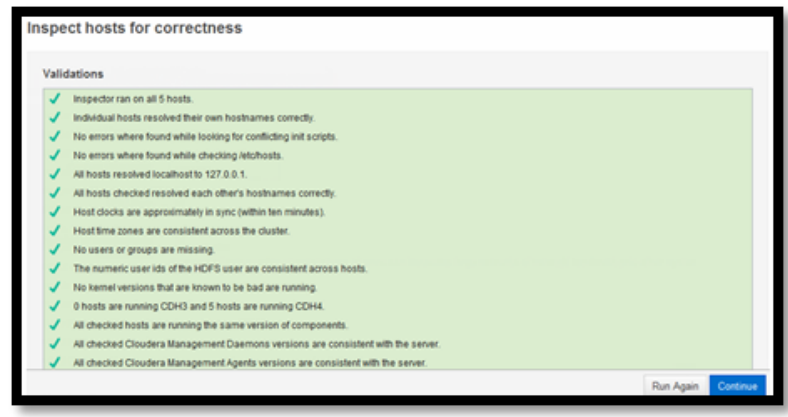

Figure 15. Services Selection Option

Figure 15 showing service we want to install on each host for CDH4

Figure 16 showing the cluster service installing steps where Cloudera manager is installing services on the nodes. 


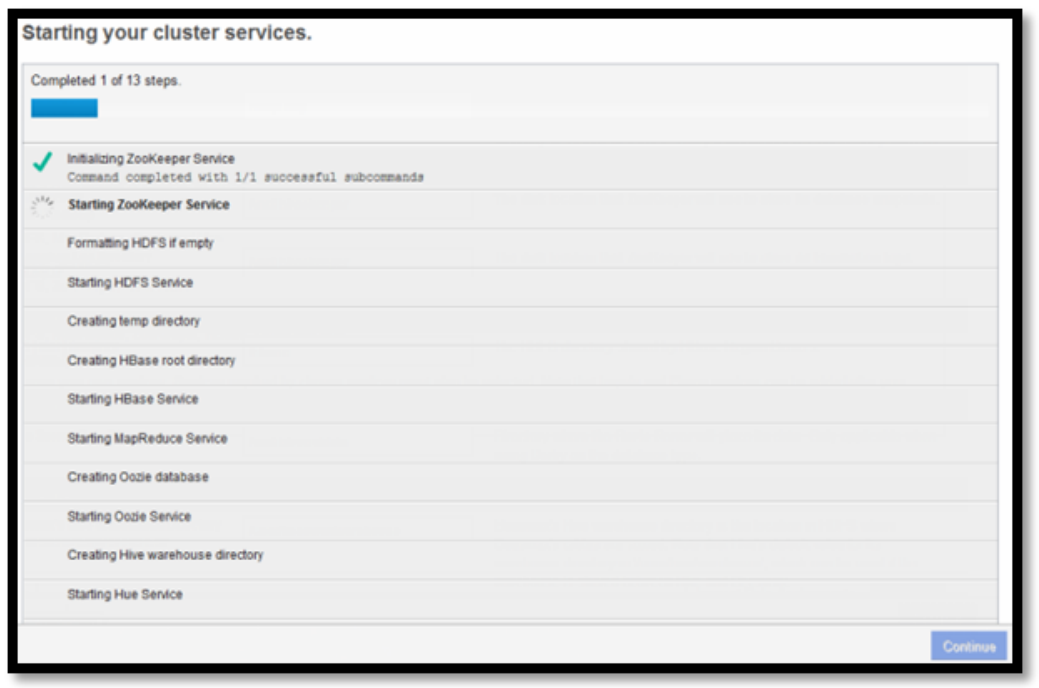

Figure 16. Cluster Service Installation

Figure 17 shows the Cloudera Manager Dashboard login in to the console through default id and password i.e. admin, admin respectively. Below screen shot shows various services of Hadoop running on the three nodes and managed by cloudera manager.

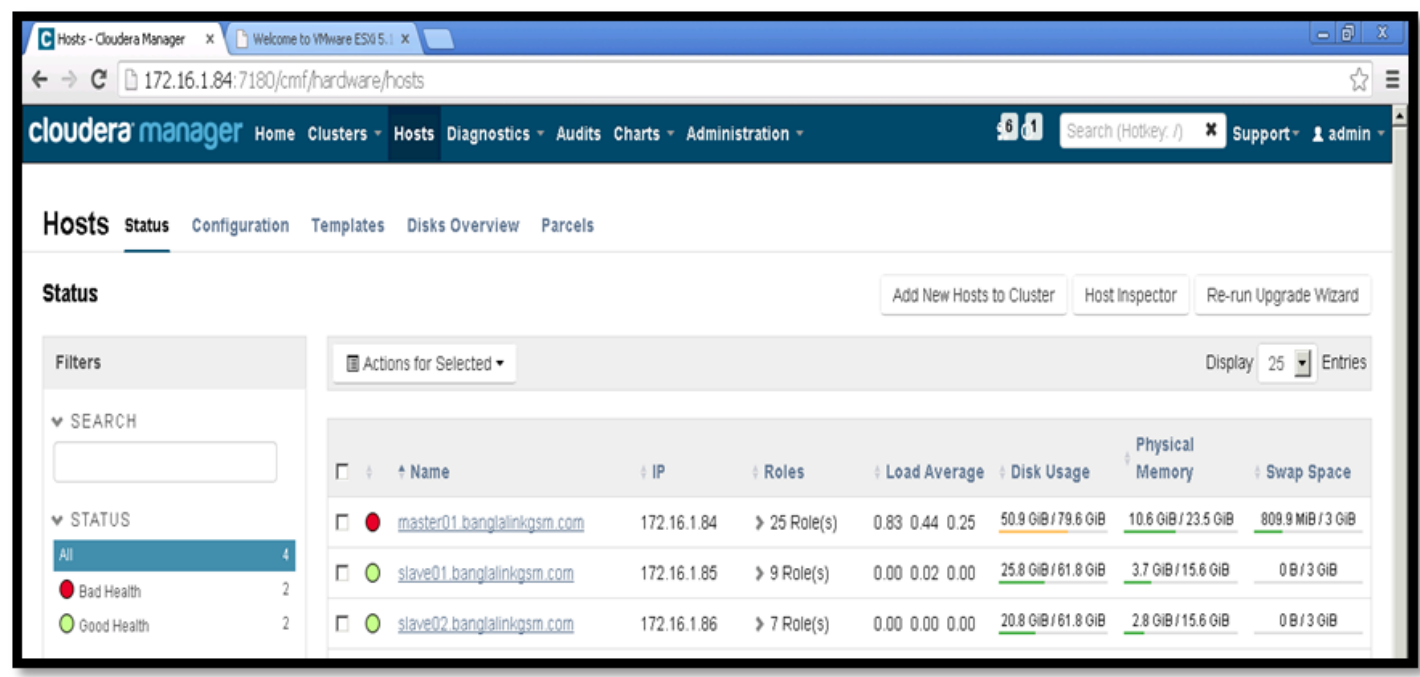

Figure 17. Cloudera Manager Dashboard

In this stage, Hadoop distribution as a Cloudera Manager CDH completely install and Hadoop cluster ready in the lab with 40 workstations for data analytics.

\section{Performance Evaluation}

With the help of TPC guideline we design Database on virtual hadoop cluster system. Generate three sets of data and table on this system. We uses TPC-DS provided Data Generator tools to generate benchmarking standard dataset.

Here Figure 3.1 shows the same dataset on a table "store_sales" on of the database Hadoop. Some queries will be execute on the table "store_sales" to compare performance of distributed parallel processing and single processing system. 


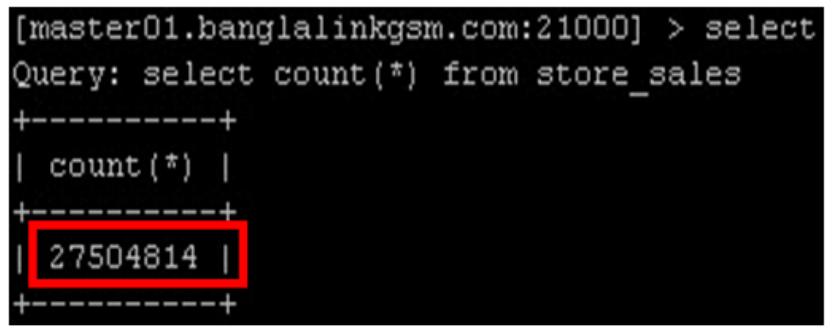

Figure 18. Hadoop Dataset

\subsection{Query Example One}

This query is collected from TPC benchmarking document query number Q3. This query report the total extended sales price per item brand of a specific manufacturer for all sales in a specific month of the year. Figure 3.3 and Figure 3.4 shows the time consumed to run the query on of the systems.

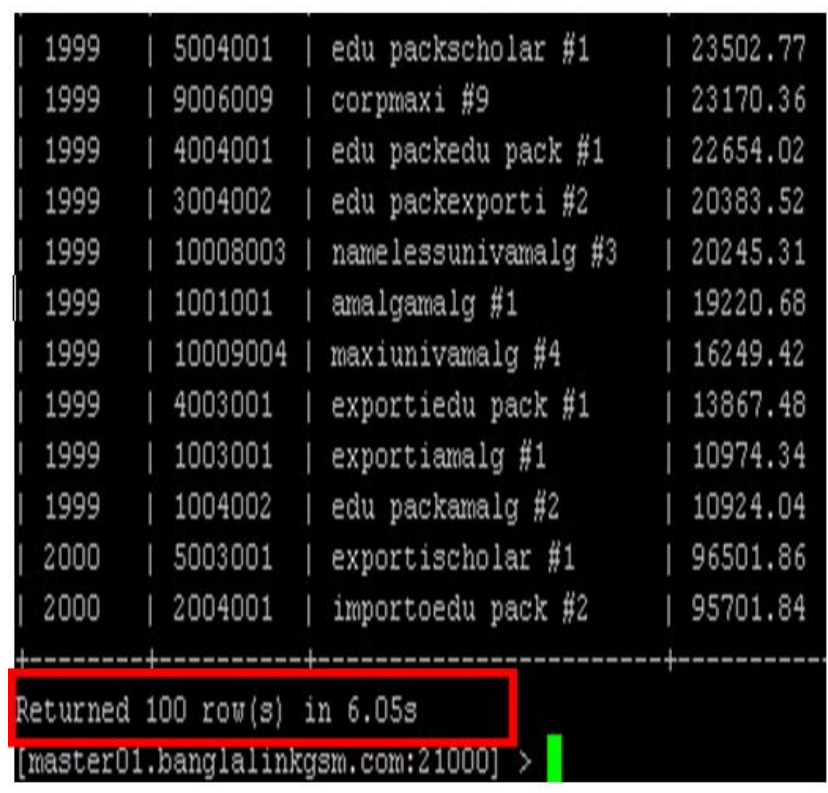

Figure 19. Q3 executed on Hadoop Cluster

\subsection{Query Example Two}

This query is collected from TPC benchmarking document query number Q7. This query report to compute the average quantity, list price, discount, and sales price for promotional items sold in stores where the promotion is not offered by mail or a special event. Restrict the results to a specific gender, marital and educational status. Figure 3.5 shows the elapsed time to process same query on same data. 


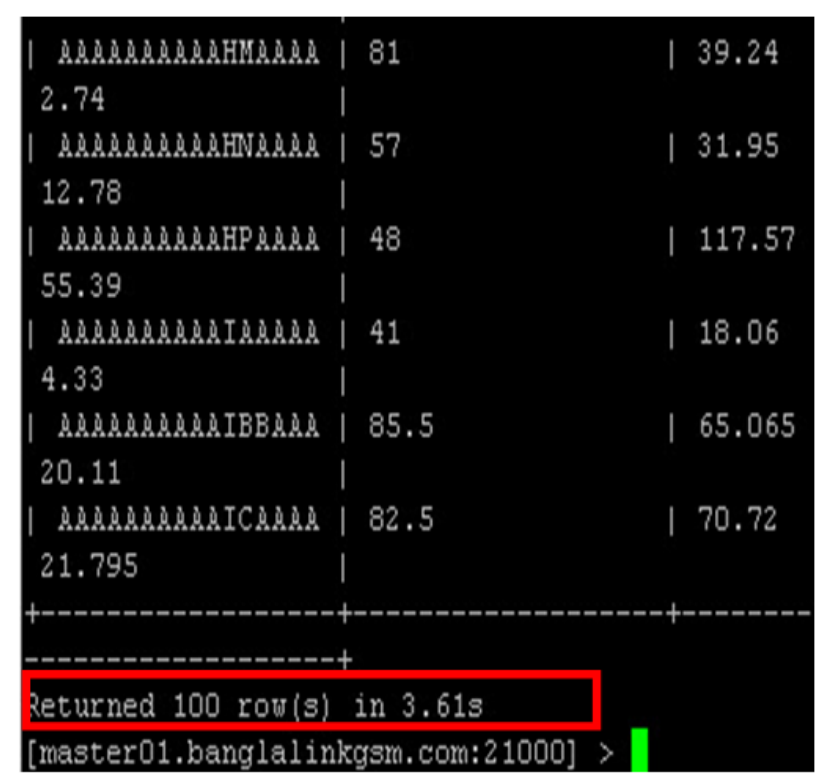

Figure 20. Q7 Executed on Hadoop Cluster

\subsection{Query Example Three}

This query is collected from TPC benchmarking document which will stress the database system by accessing all table and will apply aggregation for in-depth analysis of the database. This type of query used to stress the database to test the performance. Figure 3.7 and Figure 3.8 shows the stress test timing of the database.

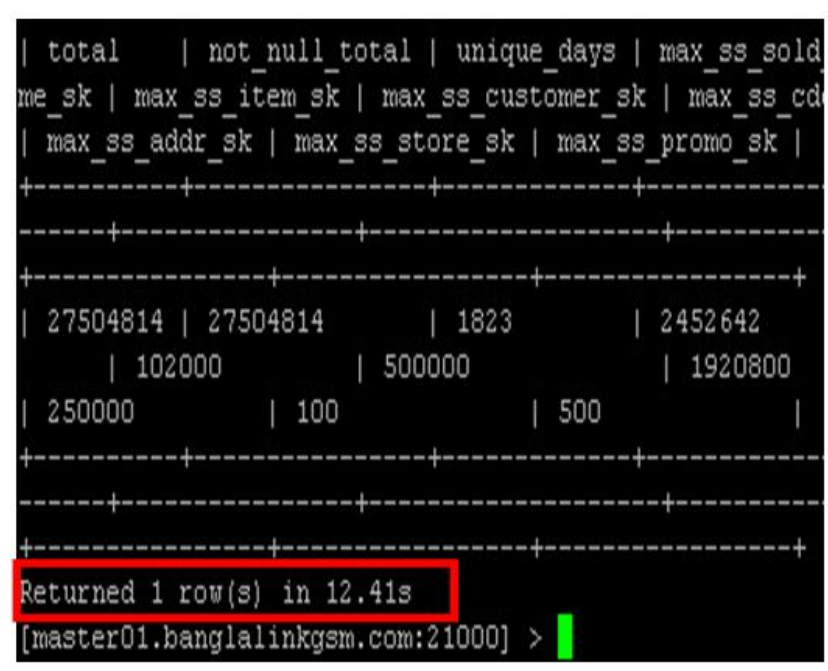

\section{Figure 21. Query Executed on Hadoop Cluster}

Here there set of experiments to investigate the performance of virtual datacenter with standard datasets and observe that data analytics easily perform in the hadoop clusters with three datasets in three different execution times. Here network bandwidth is the vital point for smooth operation of virtual cluster as well as bottlenecks of virtual cluster performance. 


\section{Conclusion}

Big data processing is currently becoming increasingly important research field in computer technology professionals due to the continuous growth of the amount of data generated by various fields. However, the processing of large-scale research data requires cluster technology infrastructure which causes huge investments for educational institutions. Hadoop is an open-source framework that allows for distributed storage and processing of very large data sets on computer clusters built from commodity hardware. This technology is being widely used for the analysis of large datasets. This paper focuses on proposes a low cost scalable hadoop virtual cluster platform and the performance of hadoop virtual cluster. We first describe the design and implementation of a virtual datacenter using hadoop framework. Then we perform a set of experiments to investigate the performance of virtual datacenter with standard datasets. For experiment and evaluation, the Cloudera's distribution of Apache Hadoop ( $\mathrm{CDH})$ is installed and configured on the DIU Cloud and Big Data Lab as a prototype implementation of a virtual data center and tested with 40 workstations.

We design Database on virtual hadoop cluster system and generate three sets of data and table on this system. Here we tried to demonstrate a performance by executing these sets of queries over the distributed parallel computing, virtual hadoop cluster system. For the simulation of the infrastructure Hadoop cluster has been used for distributed parallel processing. In according to benchmarking standard, we use TPC BENCHMARK TM DS" by-Transaction Processing Performance Council (TPC). The contributions of this paper, is to design model and implement a cost effective elastic virtual data center with hadoop framework and resource utilizations for educational institutions to provide high performance for distributed and parallel processing; as well as, Identify the bottlenecks of this systems e.g. bandwidth of network connectivity with all nodes in the lab.

\section{References}

[1] M. James, "Big data: The next frontier for innovation, competition, and productivity", (2011).

[2] M. Andrew, "Big data", the management revolution. Harvard Bus Rev, vol. 90, no. 10, (2012), pp. 6167.

[3] Z. Paul and C. Eaton, "Understanding big data: Analytics for enterprise class hadoop and streaming data", McGraw-Hill Osborne Media, (2011).

[4] https://hadoop.apache.org/

[5] https://en.wikipedia.org/wiki/Apache_Hadoop

[6] W. Tom, "Hadoop: The definitive guide", O'Reilly Media, Inc., (2012).

[7] S. Konstantin, "The hadoop distributed file system", Mass Storage Systems and Technologies (MSST), 2010 IEEE 26th Symposium on. IEEE, (2010).

[8] B. Dhruba, "The hadoop distributed file system: Architecture and design", Hadoop Project Website, 11.2007, (2007), pp. 21.

[9] D. Jeffrey and S. Ghemawat, "MapReduce: simplified data processing on large clusters", Communications of the ACM, vol. 51, no. 1, (2008), pp. 107-113.

[10] D. Jeffrey and S. Ghemawat, "MapReduce: a flexible data processing tool", Communications of the ACM, vol. 53, no. 1, (2010), pp. 72-77.

[11] http://www.cloudera.com/content/cloudera/en/home.html

[12] http://www.cloudera.com/content/cloudera/en/products-and-services/cdh.html

[13] M. Hossen, A. K. M. Moniruzzaman, A. B. M. and Hossain S. A., "Performance Evaluation of Hadoop platform and Oracle for Distributed parallel Processing in Big Data Environments arXiv preprint arXiv", (2015). 
Authors

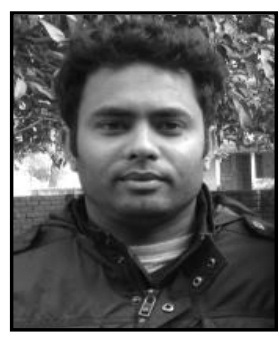

Seraj Al Mahmud Mostafa, is an M. Sc. graduate in Computer Science and Engineering from Lulea University of Technology, Sweden. He is currently working as a lecturer at Daffodil International University, Dhaka, Bangladesh. Besides this, he is involved in a Data Mining and Big data analysis research project at this university under Computer Science research team. His research papers and technical reports can be found in IEEE explore and the Lulea University research database. He had also been in the organizing committee for two conferences in Lulea University of Technology, Sweden. His research areas include Big Data Analytics, Hadoop, Data mining, Open-source Clouds, Cloud Management Platforms, Data Engineering and Management, and privacy issues for online users.

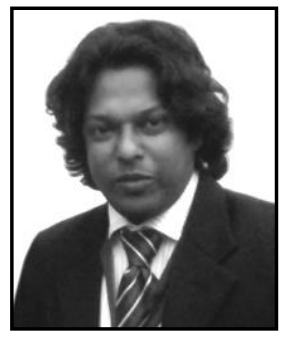

A B M Moniruzzaman, is a $\mathrm{PhD}$ researcher at Jahangirnagar University; he received his B. Sc. (Hon's) degree in Computing and Information System (CIS) from London Metropolitan University, London, UK and M. Sc. degree in Computer Science and Engineering (CSE) from Daffodil International University, Dhaka, Bangladesh in 2005 and 2013, respectively. Currently he is working as a Lecturer of the department of Computer Science and Engineering ad Daffodil International University. His voluntarily works as reviewer of many international journals including IEEE, Elsevier, Springer, IGI-Global, InderScience. Besides, he has been serving as Technical Program Committee Members in many (over nineteen) different international Conferences. He has 13 (thirteen) international publications including journals and conference proceedings. He is a member of IEEE since 2012. He has been serving as a counselor DIU IEEE STUDENT BRANCH. His supervision project 'Solar Car' has received international award and funded by ICT innovation fund by Govt. of People's Republic of Bangladesh. His research interests include Cloud Computing, Cloud Applications, Open-source Clouds, Cloud Management Platforms, Big Data Management, Agile Software Development, Hadoop, MapReduce, Parallel and Distributed Computing, Clustering, High performance computing, Distributed Databases, NoSQL Databases. 
International Journal of Database Theory and Application Vol.8, No.6 (2015) 